This is the author's final, peer-reviewed manuscript as accepted for publication. The publisher-formatted version may be available through the publisher's web site or your institution's library.

\title{
Cells as delivery vehicles for cancer therapeutics
}

Matthew T. Basel, Tej B. Shrestha, Stefan H. Bossmann \& Deryl L. Troyer

\section{How to cite this manuscript}

If you make reference to this version of the manuscript, use the following information:

Basel, M. T., Shrestha, T. B., Bossmann, S. H., \& Troyer, D. L. (2014). Cells as delivery vehicles for cancer therapeutics. Retrieved from http://krex.ksu.edu

\section{Published Version Information}

Citation: Basel, M. T., Shrestha, T. B., Bossmann, S. H., \& Troyer, D. L. (2014). Cells as delivery vehicles for cancer therapeutics. Therapeutic Delivery, 5(5), 555-567.

Digital Object Identifier (DOI): doi:10.4155/tde.14.24

Publisher's Link: http://www.future-science.com/doi/abs/10.4155/tde.14.24

This item was retrieved from the K-State Research Exchange (K-REx), the institutional repository of Kansas State University. K-REx is available at http://krex.ksu.edu 


\title{
Cells as Delivery Vehicles for Cancer Therapeutics
}

\begin{abstract}
Cell-based therapeutics have advanced significantly over the past decade and are poised to become a major pillar of modern medicine. Three cell types in particular have been studied in detail for their ability to home to tumors and to deliver a variety of different payloads. Neural stem cells, mesenchymal stem cells and monocytes have each been shown to have great potential as future delivery systems for cancer therapy. A variety of other cell types have also been studied. These results demonstrate that the field of cell-based therapeutics will only continue to grow.
\end{abstract}

\section{Defined Key Words}

Tumor tropism - the ability of certain cells to selectively home to and infiltrate tumor tissues

Chemotaxis - the ability of certain cells to respond to concentration gradients in order to migrate up or down gradient.

Magnetic hyperthermia - a method for producing therapeutic hyperthermia that utilizes magnetic nanoparticles to absorb radio-frequency radiation and convert the energy to heat. This allows hyperthermia to be targeted to locations containing the magnetic nanoparticles, which, when combined with cell-delivery, may prove to be a highly focused tumor treatment.

Cell-based gene therapy - cells are engineered with a therapeutic gene which can then be expressed in the tumor tissue when the cells migrate to the tumors.

Suicide gene - a gene whose product is a prodrug activating enzyme that converts a relatively non-toxic prodrug into a toxic drug causing the cell to commit suicide. In cell-based cancer therapy this can be used either for bystander effect to kill tumor cells and/or as a way to eliminate delivery cells after they have exhausted their usefulness.

\section{Executive Summary}

Introduction

- Cells have been shown to be a potentially valuable targeting method.

- Cells have advantages over other targeting methods because of their active targeting abilities and the wide variety of cargos they can deliver.

Neural Stem Cells

- Since the first report in 2000 , neural stem cells have been used extensively in preclinical models as delivery cells for tumor treatment 
- Neural stem cells have been used primarily to target glioma/glioblastoma, but can be used to target other tumors.

- Neural stem cells are particularly useful for providing gene therapy, but can also be loaded with other therapeutic materials such as nanoparticles.

Mesenchymal Stem Cells

- Due to their strong tumor tropism, mesenchymal stem cells have become another major focus of cell-directed tumor treatment.

- Highly versatile cells, MSCs can deliver a wide variety of therapies.

- Some results have shown a natural tumoricidal nature to the MSCs, although many studies have shown tumor promoting properties.

Monocytes

- Highly-abundant, easily-obtainable, patient-specific, and tumor-tropic, monocytes are a third focus of cell-directed therapy.

- Monocytes are particularly adept at delivering nanoparticle treatments, but can be used for other therapies.

Other Cells

- Several other cell types have also been tested as delivery cells for tumor therapy.

- Using T cells to deliver chimeric antigen receptors are particularly intensively being investigated.

\section{Introduction}

Cell-based therapeutics appear poised to join small-molecule drug discovery and biologics as a major arena of modern medicine[1], highlighted by recent publication of major works in this field [2]. In contrast to the first two pillars (small molecules and biologics), cells are able to sense a variety of signals, travel to specific points in the body, actively penetrate areas of pathology, and deliver a therapy to minimize off-target effects.

Since various stem and defensive cells have been convincingly demonstrated to traffic to tumors, they offer the potential of more tumor specific therapies that avoid the many off-target effects seen with conventional chemotherapy. These cells, in contrast to many other strategies such as nanoparticles that rely on passive accumulation, can infiltrate the tumor after they access it despite the elevated interstitial pressure and formidable stromal barrier inherent in most tumors (figure 1). The cells are attracted to tumors by gradients of chemokines, cytokines, and growth factors. Examples include VEGF, MCP1, the CXCR4-CXCL12 system [3], and transforming growth factor beta (TGF- $\beta$ ) [4].

Cells can be utilized to deliver a wide variety of different payloads, including small molecule drugs, proteins, suicide genes, nanoparticles, viruses, etc. and some cell types have been shown to be naturally tumoricidal. Although other platforms may be able to deliver these payloads, cells have advantages over many other platforms in being able not only to deliver but also to 
continuously produce many of their payloads after arrival onsite. Examples include therapeutic proteins (after genetic engineering the delivery cell), suicide genes or viruses and even nanomaterials..

Here we summarize some of the recent developments in the field of cytotherapy for cancer focusing on three oft-used cell types: neural stem cells, mesenchymal stem cells and monocytes as well as several other types which will be briefly reviewed at the conclusion of the review. The three major cell types have been extensively researched over the past decade demonstrating that cell-based therapeutics may soon become a staple of cancer therapy.

\section{Neural Stem Cells}

It was the pioneering work of Aboody and colleagues in 2000 that ignited enormous interest in the paradigm of using cells as targeting vectors to treat cancer [5]. They showed that human neural stem cells (NSCs) transplanted into either the ipsilateral or contralateral cerebral hemisphere of mice bearing intracranial gliomas could with high fidelity migrate into the tumors. In fact, the NSCs appeared to be also trailing aggressive glioma cells that had migrated from the main tumor mass. The NSCs were engineered to express cytosine deaminase, and when the prodrug 5 fluorocytosine $(5-\mathrm{FC})$ was administered, the homing cells could convert it to 5-FU (5-fluoro-uracil), an anticancer drug with resultant attenuation of glioma growth.

Subsequent to this work, many papers showing the efficacy of using neural stem or progenitor cells for targeted delivery of therapeutic agents have appeared. It was shown that intravenously administered NSCs could deliver a secreted prodrug activating enzyme, rabbit carboxylesterase, to a mouse model of metastatic neuroblastoma [6]. After administration of the prodrug irinotecan, survival time was dramatically increased.

Neural stem cells have also been used to deliver other suicide genes into tumors. NSCs engineered with a cytosine deaminase gene (used to deaminate 5-fluorocytosine to the toxic 5fluorouracil) were used to target the suicide gene to medulloblastoma [7]. The engineered NSCs were shown to migrate well into the medulloblastomas similar to previous glioma studies. After treatment with 5-flourocytosine, the NSC treated medulloblastoma-bearing mice showed significant increases in survival time compared to controls. Follow up work showed that adding a second suicide gene, thymidine kinase, to the cytosine deaminase-bearing NSCs can improve the treatment effect [8]. A mouse model of metastatic lung cancer in the brain treated with the dual-suicide gene-bearing NSCs and both prodrugs, 5-fluorocytosine and ganciclovir, showed both significant survival increases over controls and significant decrease in tumor volume.

Because the blood brain barrier (BBB) limits access of chemotherapy drugs to glioma, and glioma cells have a pronounced tendency to develop chemoresistance, cell based delivery is particularly attractive for this type of neoplasia [9]. NSCs given systemically can traverse the $\mathrm{BBB}$, although this route is not very efficient.

Another rather surprising route for stem cell mediated delivery to glioma that can avoid the BBB has recently emerged - the nasal cavity. Neural stem cells were administered intranasally 
to mice bearing U87, NCE-G55T2 human tumors or syngeneic mouse GL261 tumors [10]. were monitored by MRI imaging, with significant accumulation at six hours and maximal accumulation at 24 hours. Most cells traveled along the olfactory tract but some were apparently distributed systemically via the microvasculature of the nasal mucosa.

NSCs engineered to make a secreted form of tumor necrosis factor-related apoptosis-inducing ligand (TRAIL) decreased glioma burden in a mouse model [11]. However, since glioma cells often develop resistance to TRAIL, various adjunct therapies including proteasome inhibitors [12] and kinase inhibitors [13] have been used to mitigate this resistance.

Neural stem cells engineered to secrete anti-tumor antibodies have been used to successfully attenuate tumor growth. For example, NSCs expressing variants of EGFR-specific nanobodies reduced glioma growth and invasiveness [14]. NSCs secreting an anti-HER2 antibody inhibited breast cancer growth in vitro and although an anti-tumor effect wasn't shown, migrated successfully to in vivo to breast cancer xenografts [15].

Neural stem cells have also been used as Trojan horses to carry oncolytic virotherapy into glioblastomas. HB1-F3-CD cells (a v-myc immortalized, FDA approved neural stem cell line) were infected with CRAd-S-pk7 virus designed to selectively replicate in glioma cells and spare whole brain toxicity [16]. Using neural stem cells to deliver the virus increased localization and concentration of the virus and minimized immune reactivity of the virus. A follow up study demonstrated that using the neural stem cells to deliver the virus also enhanced the therapeutic efficacy of the virus [17]. The neural stem cells were also shown to deliver the virus better than mesenchymal stem cells [18]. In addition, the virus-loaded neural stem cells can be combined with standard of care treatments for glioblastoma. By correctly timing delivery of virus-loaded cells a significant increase in median survival was demonstrated in mice bearing human xenograft gliomas over treatment with only radiation and temozolomide [19].

NSCs preloaded with gold nanoparticles have been used as carriers to deliver photothermal ablation of MDA-231 breast cancer cells and tumors (although in the in vivo study the group size of two was too small for statistical evaluation) [20].

Our group has demonstrated that neural stem cells can be used to deliver $\mathrm{Fe} / \mathrm{Fe}_{3} \mathrm{O}_{4}$ nanoparticles to a mouse model of melanoma for inducing hyperthermia in alternating magnetic fields (figure 2) [21]. Loading with $\mathrm{Fe} / \mathrm{Fe}_{3} \mathrm{O}_{4}$ showed little toxicity to the neural stem cells and did not prevent their trafficking to subcutaneous melanomas when injected intravenously. The delivery of nanoparticle-loaded neural stem cells alone had a small but measurable tumoricidal effect. When treated with alternating magnetic fields in order to induce magnetic hyperthermia in the tumors using the nanoparticles, an increased tumoricidal effect was demonstrated that was significantly different than control. In vitro studies demonstrated the ability of the nanoparticle-loaded neural stem cells to significantly increase the temperature in the tumor leading to hyperthermia effects.

It is not practical to derive patient specific NSCs since they are only located in a few sites deep within the brain. However, it has been reported that it is possible to obtain induced neural 
stem cells (iNSCs) by the introduction of the Sox2 gene into fibroblasts [22]. This could offer a shortcut to the derivation of NSCs from induced pluripotent stem cells (iPS cells), which has been reported to also offer the prospect of patient-specific NSCs. For example, NSCs derived from iPS cells have been shown to migrate to models of breast cancer similar to their natural counterparts and can deliver suicide gene therapy to the tumors [23]. The migration of the iPSderived NSCs was shown to be further increased by inhibiting neuronal nitric oxide synthase through either small molecule inhibitors or siRNA providing a pathway for increasing tumor tropism [24]. Human neural stem cells that are in clinical trials [25] are of fetal origin and are not patient specific. However, it is thought that NSCs are relatively non-immunogenic [26], in fact, they may suppress immune response [27]. Recently, an agenetic approach to transdifferentiating CD34+ bone marrow cells into neural progenitors has been described which may provide another pathway for generating patient-specific neural stem cells [28]. Donorderived CD34+ cells were treated with G-CSFR binding antibodies and compared to G-CSF treated CD34+ cells. As opposed to the G-CSF treated cells, which merely proliferated, the antiG-CSFR antibody-treated cells demonstrated morphologies associated with neural cell lineages. Analysis showed the presence of Tuj1 and nestin, which are neural markers. Although the exact mechanism of transdifferentiation is unknown, it was postulated that it may have to do with not only the signal-receptor interaction but also the intensity and duration of interaction that caused the G-CSFR receptor to cause transdifferentiation. This may provide a source of easily extractable, patient-derived neural stem cells for future treatments.

Thus, neural stem cells offer enormous opportunity for cell-based, targeted therapy of glioblastoma and other types of cancer. An especially exciting attribute is their ability to follow invasive cells that would otherwise escape surgical resection. A clinical trial (NCT01172964) is underway to evaluate a human NSC cell line engineered to secrete the prodrug activating gene cytosine deaminase, a nucleotide salvage enzyme derived from E Coli, for glioma therapy. The use of NSCs to carry oncolytic viruses to glioma not only can better target the virotherapy, it can also avoid the immune system.

However, long term consequences of using immortalized stem cells remain to be addressed. If the cells remain after tumor resolution will the gene therapy damage normal tissue? Will they themselves be prone to tumor development at some later date? Utilizing cells engineered to express a suicide gene should confer a level of safety assuming the cells themselves will eventually succumb. Another issue is that the most efficient route of administration of NSCs for glioma therapy is intracranial inoculation, which is invasive.

\section{Mesenchymal Stem Cells}

Soon after Aboody and colleagues showed neural stem cells could home to and deliver an anticancer therapy to glioma, it was reported by Studeny et al. that bone marrow mesenchymal stem cells (BMSCs) engineered to express beta interferon (IFNb) could home to metastatic lung melanoma [29]. This study has led to the development of a robust research area in tumor 
tropism of mesenchymal stem cells MSCs combined with delivery of therapeutic or prodrug activating gene delivery for cancer therapy [30-32].

In 2007, our group showed that umbilical cord mesenchymal stem cells derived from Wharton's jelly (UCMSCS) could be used to deliver a cytokine to MDA-MB-231 breast cancer pulmonary metastases [33]. The local delivery of beta interferon significantly attenuated the metastatic tumors. When the UCMSCs expressing IFNb were combined with low dose 5-fluorouracil (5FU), there was an additive anti-tumor effect [34].

UCMSCs can have a potent intrinsic anti-cancer effect. When naïve (unmodified) rat UCMSCS were administered intravenously to Fisher 344 rats bearing mammary carcinomas, the tumors were completely eradicated with no recurrence [35]. In vitro colony assay showed that the anti-cancer effect of rUCMSCs is due to increase in caspase-3 activity and an antiproliferative effect on Mat B III cells (mammary carcinomas).

MSCs from various sources have been shown to have immune- privileged status [36]. This attribute may allow their use as off-the-shelf allogeneic cell transplants after expansion and/or modification.

BMSCs have been utilized for cell based therapy of glioma; they cross the BBB effectively and can be readily obtained from a variety of sources. IFN-beta expressing (engineered) BMSCs migrated to U87 glioma tumors, and significantly increased the survival of tumor bearing nude mice compared with control [37].

There is tremendous interest in using cells to deliver immunotherapy to tumors. BMSCs were engineered to express tumor necrosis factor superfamily 14 (TNFSF-14; a.k.a. LIGHT) [38]. The LIGHT expressing MSCs homed effectively to tumors and organized a potent anti-tumor immune therapy by stimulating an influx of T cells. In another study umbilical cord blood derived MSCs were engineered to express a secretable form of LIGHT and attenuated subcutaneous tumors in a model of human gastric cancer [39].

Other papers have shown the efficacy of using MSCs as carriers of oncolytic viruses [40,41]. This strategy not only results in better targeting of the virus to the tumor, but it shields the virus from the immune system. Mader et.al showed that MSC can shield Measles Virus and successfully deliver measles virus to tumor-bearing mice, showing significantly increased the survival of mice compare with naked $\mathrm{M}$ virus. Replication-competent oncolytic adenovirus (CRAd) loaded hMSC migrated to distant glioma tumors and delivered more viral particles than distance injection of viral particle alone [42]. Upon i.p. administration of MSC containing oncolytic viruses particles (Ad5/3, coxsackie and adenovirus receptor-independent infection) the cells homed to the ovarian cancer in a mouse model, decreased tumor burden and increased the survival of mice compared with direct administration of viruses [43].

MSCs have been used as carriers to deliver therapeutic nanoparticles to tumors or tumor spheroids. Roger et al showed that Coumarin-6 loaded polylactic acid NPs (PLA-NPs) and lipid nanocapsules (LNCS) can be efficiently loaded to MSCs without affecting cells, and these loaded 
cells migrate toward tumors in a human glioma model in nude mice [44]. They also validated that lipid nanocapsule (LNCS) could be loaded without any toxic effect to the delivery cells (mesenchymal stem cells; MSCs) and became distributed around the glioma cells after intratumoral injection of these LNCs loaded cells. In vivo experiments showed that intratumoral injection of ferrociphenol (Fc-diOH)-LNC-loaded delivery cells to orthotropic glioma in nude mice showed cytotoxic effect [45].

MSCs have also been used to deliver INF-beta to ovarian tumors. MSCs were engineered to secrete INF-beta and were injected intraperitoneally in mice bearing syngeneic or xenograft tumors. These MSCs were shown to infiltrate the ovarian tumors, and complete eradication of tumors was observed in up to $70 \%$ of cases with increased survival in treated mice [46].

Adipose tissue derived MSCs (ADMSCs) have been used to deliver various therapies to tumors. For example, AD-MSCs expressing yeast cytosine deaminase::uracil phosphoribosyltransferase (CD::UPRT) in combination with 5-FC efficiently suppressed subcutaneous (s.c.) human colon cancer tumors in nude mice [47]. These ADMSCs expressing yeast CD::UPRT/5-FC also could attenuate intracranial $\mathrm{C} 6$ rat glioblastoma [47]. The same group showed that these cells engineered with the same prodrug activating enzyme could attenuate bone metastatic prostate tumors in a mouse model not only when the ADMSCs were co-injected with the prostate cancer cells, but more importantly, when those cells were delivered in a cell dose dependent manner intravenously [48]. They also found that co-injection of $20 \%$ these cells with human melanoma cells could render 8 out of 9 mice tumor free. Moreover, intravenous injection of these cells with subsequent administration of prodrug reduced the tumor size of s.c. melanoma in a mouse model [49]. In fact, it was found that this cell therapy could attack cancer stem cells, thus being a curative therapy for a rodent model of glioblastoma [50]. Another group showed that TRAIL engineered AD-MSCs given I.V. to a rodent model of cervical carcinoma could selectively deliver a proapoptotic ligand to the tumor site without side effects. It also eliminated the problem of low stability of TRAIL in blood plasma [51].

Naïve ADMSCs were shown to promote human melanoma tumor growth but had the opposite effect in glioblastoma [32]. Naïve BMSCs also have been shown to have an antitumoral effect on an animal model of Kaposi's sarcoma [52]. However, there are other reports indicating that naïve BMSCs promote tumor growth [53]. Both BMSCs and Adipose derived MSCs have been shown to differentiate into cancer associated fibroblasts (CAF) which are known to promote tumor growth $[54,55]$.

There have been a few cautionary results with mesenchymal stem cells. AD-MSCs also migrate to prostate cancer. Lin et al. showed that these cells migrated to s.c. PC3 prostate cancer in athymic mice although they increased the tumor size by increasing the tumor vascularity, potentially limiting their clinical usefulness [3]. Similarly, bone marrow derived MSCs migrate to multiple myelomas through attraction to the cytokine CCL25 and promote tumor growth through a variety of complex interactions [56], although contradictory results demonstrate that MSCs can prevent relapse and encourage bone formation in multiple myeloma models [57]. AD-MSCs also migrate to U87 tumors in mice (intracranial gioma xenograft). Intratumorally or 
contralaterally injected PKH 26 labeled AD-MSCs home to U87 tumor in mice, but adenovirus infected AD-MSCs didn't migrate to the same tumor [58].

Because mesenchymal cells are primary cells with unstable life times, generation of suitable numbers for therapy can be difficult. Recently, immortalized bone-marrow derived MSCs have been shown to continue to home to prostate tumors and to deliver a thymidine kinase suicide gene. No harmful side effects, particularly no increase in lymphocytes were noted in the treated mice indicating that immortalized MSCs may provide a more stable and abundant source of MSCs for therapeutic use.[59] Although AD-MSCs have not been immortalized, studies have shown that expanding AD-MSCs in culture provide stable and non-tumorgenic MSCs for use in therapy [60].

Taken together, these data characterize MSCs as suitable delivery vehicles for prodrug converting gene and show their utility for a personalized cell-based targeted cancer gene therapy. It is concerning that bone marrow or adipose derived naïve MSCs can enhance tumor growth, so anti-tumor therapies must overcome this effect. All reports of human or rodent umbilical cord MSCs to date indicate the naïve cells themselves attenuate tumors. However, this advantage of the latter cells may be offset by the fact that they are not autologous cells.

\section{Monocytes}

Monocytes are known to infiltrate most solid tumors where they usually become $\mathrm{M} 2$ macrophages which are pro-tumorigenic [61-63]. M2 macrophages are associated with tissue healing and express a variety of anti-inflammatory signals. These signals promote tumor growth especially through suppressing T-cell response through recruitment of T-regulatory cells (Tregs) through CCL22 and suppression of T cytotoxic cells through expression of arginase and other immune modulators. Also, M2 macrophages can promote angiogenesis, tissue remodeling for invasion, and have a protective effect against chemotherapeutic damage. Since these cells have a pronounced tumor tropism they are good candidates for delivery of therapeutics or ex vivo treatment to differentiate them into tumoricidal macrophages [64-67]. The cells can be easily isolated in large numbers from peripheral blood to be modified ex vivo as autologous cellular therapy. Tie-2 expressing monocytes are particularly tumor tropic cells[6870], and Tie-2 expressing monocytes can be used to deliver therapy selectively to tumors [71]. Thus monocytes have potential to be valuable delivery vehicles for cytotherapy.

Although monocytes traffic to the hypoxic regions of most solid tumors, there are ways to improve this migration. Chemo or radiation therapy can attract additional monocytes because of the tissue damage caused by the treatment [72-76]. Monocytes cultured in hypoxic environments upregulate CXCR4 expression, which increases homing along the CXCL12 gradient often present in tumors [77]. It could also be possible to anchor a tumor-homing peptide to the delivery cell wall to enhance migration. This strategy was recently reported to increase migration of MSCs to myocardial infarcts (MI) in mice [78]. Although the peptide was identified by phage display to target MI regions, similar peptides have been identified for tumor 
homing [79], so theoretically the concept could be applied to enhance tumor migration. In this paradigm, the peptide was a palmitated derivative that could be used to attach to the plasmalemma to coat the delivery cells. Finally, magnetic fields have been used to attempt to increase monocyte trafficking into tumors [80]. By loading monocytes with magnetic nanoparticles, a properly placed magnet can increase their migration into tumor spheroids in vitro, although the applications of this in vivo have yet to be shown.

Monocytes can be loaded ex vivo with nanoparticle payloads similar to what has been shown for neural stem cells and neural progenitor cells (see above). They have been loaded, for example, with gold nanoparticles for active delivery of this cargo into tumors so NIR radiation can be used to induce photothermal damage [81]. Later it was shown that these nanoparticleloaded monocytes can infiltrate as well or better into glioma spheroids in vitro. Treatment with NIR radiation in the presence of loaded monocytes can completely attenuate spheroid growth [82]. Our group has used monocyte-like cells to deliver iron/iron oxide nanoparticles to pancreatic tumors in a mouse model of disseminated pancreatic cancer. We demonstrated that Raw264.7 cells, a monocyte/macrophage-like cell line, can traffic selectively to pancreatic tumors disseminated in the peritoneal cavity while sparing healthy pancreatic tissue when the Raw264.7 cells were injected intraperitoneally (figure 3). Raw264.7 cells also take up core/shell iron/iron oxide nanoparticles without significant toxicity and can thus be used to deliver the nanoparticles to the pancreatic tumors. The nanoparticles can then sensitize the tumors to alternating magnetic frequency radiation (AMF) inducing magnetic hyperthermia selectively in the tumor tissue. Treatment with nanoparticle-loaded Raw264.7 and AMF radiation significantly increased the survival time of mice bearing disseminated pancreatic tumors [83].

Monocyte/macrophages can also be used to deliver gene products into tumors. Our group has used Raw264.7 to deliver a self-contained suicide gene-prodrug combination to a model of metastatic melanoma in the lungs. A TetOn ${ }^{\circledR}$ system for expressing rabbit carboxyesterase (RCE) was stably transfected into Raw264.7 cells that causes expression of RCE upon treatment with a tetracycline such as doxycycline. RCE can efficiently activate the prodrug irinotecan to SN38. These cells were demonstrated to traffic to melanomas in the lungs. The cells were loaded with irinotecan and injected intravenously into mice with metastatic melanoma. When treated with both the loaded cells and doxycycline the treatment decreased the number, weight and size of melanoma tumors in the lungs as compared to controls [84].

In a follow up study, the same TetOn ${ }^{\circledR}$ RCE cells were used to deliver RCE and an SN38 prodrug to a model of disseminated pancreatic cancer. Combining the concept of delivering nanoparticles and delivering a chemotherapy drug, an irinotecan-like prodrug was synthesized on iron/iron oxide nanoparticles in order to increase loading and retention in the Raw264.7 cells. Using these cells to deliver the SN38 prodrug and treating with doxycycline significantly increased survival in a model disseminated pancreatic cancer [85].

Monocytes can also be used for virotherapy. Monocytes have been used to deliver replication competent oncolytic adenoviruses to pancreatic tumors, although the greatest effect was with monocytes injected intratumorally rather than intravenously, raising doubts as to whether 
these cells homed well to the tumors [86]. Similarly, monocytes have been used to deliver adenovirus designed to replicate only in hypoxic conditions to prostate tumors. This study demonstrated that the monocytes can be given systemically and still achieve specificity of infection in the tumor [87].

Closely related to monocytes, macrophages have also been utilized for targeting cancer therapy. Macrophages have been used to deliver nanoparticles across the blood-brain barrier into metastatic breast cancer in the brain [88]. Both peripheral monocytes and peritoneal macrophages have been used to deliver oligomannose-coated liposomes to metastatic gastric cancer.[89] Peritoneal macrophages have also been used to deliver liposomal doxorubicin to both subcutaneous and metastatic models of lung carcinoma [90]. Batrakova and colleagues have reported that macrophages can be loaded ex vivo with a self-assembled catalase/PEI-PEG complex, penetrate the blood brain barrier, and deliver the catalase to diseased neurons in an Parkinson's disease model [91]. A similar approach could be potentially used to deliver a prodrug activating enzyme or other protein therapy to tumors without introducing a foreign gene into the homing cells.

One benefit of using monocytes (as opposed to neural stem cells or mesenchymal stem cells) is that they can be easily isolated in large numbers as patient specific cells from peripheral blood. Another advantage may be their naturally limited lifespan. Monocytes differentiate to macrophages in tissues, with lifespans in the range of months [92]. Because of their expected termination, there are fewer worries about persistence and continued release of anti-cancer agents into normal tissue. Other benefits of monocyte therapy include the relative ease of obtaining autologous monocytes (from blood) or macrophages (peritoneal lavage) and their natural phagocytic nature that allows them to be loaded easily with imaging or therapeutic nanomaterials. On the other hand, monocytes can be difficult -to-transfect with plasmid DNA, making cell-delivered gene therapy relatively more difficult than with other possible cell types. Also, since monocytes normally differentiate into M2 macrophages in the tumor microenvironment they pose a danger of promoting tumor growth unless steps are taken to block this outcome. Therefore, although monocytes engineered to express various genes have been used to treat tumors, their greatest potential may be in delivering nanoparticle therapies which can be easily loaded and delivered to the tumor site.

\section{Other Delivery Cells}

Endothelial progenitor cells (EPCS) or endothelial cells have been shown to home effectively to tumors and have been utilized to deliver anti-cancer therapeutics. For example, it was shown that human EPCs isolated from peripheral blood could be engineered to express thymidylate kinase, administered IV to mice bearing subcutaneous human glioma tumors and regress the tumors upon administration of ganciclovir [93].

Myeloid-derived suppressor cells (MDSC) are immature granulocyte or monocyte cells recruited from the bone marrow in tumor bearing hosts. It has recently been shown that these cells can 
deliver an oncolytic virus to mouse models of hepatic and lung metastases [94]. Interestingly, the anti-tumor effect was due not only to the oncolytic virus but activation of the monocytic suppressor cells to the M1 phenotype.

Another specialized field of cell-directed therapy against tumors is chimeric antigen receptor (CAR) expressing T-cells. Although this review does not comprehensively cover this large field, some current work in this field should be mentioned. Early work in the late 1980s demonstrated that the natural cytotoxicity of T-cells could be harnessed and directed against tumors through modifying them to express a tumor-selective, single-chain variable fragment antibody attached to the CD3 $\zeta$ trans- and endo-domains [95-98]. Since the initial discovery, CAR has been intensively studied and several iterations of the original gene have been proposed. Second generation CAR utilizes not only the primary signal of CD3 $\zeta$ but also incorporates a costimulatory signal such as CD28. Recently third generation CAR technology continues to expand on this by incorporating multiple costimulatory signals to increase activation and proliferation of the tumor specific T-cells. For example, incorporation of CD137 signaling domain in the CAR showed an increase in antitumor activity in the hematologic malignancies. Human T cells engineered with anti CD19 with CD 137 survived longer than 6 months in vivo and were highly effective on human pre-B-cell acute lymphoblastic leukemia in a mouse xenograft model and this combination was significantly more effective than T-cells expressing T cell receptor-zeta (TCR- $\zeta$ ) alone or CD28- $\zeta$ signaling receptors [99]. The same group later on showed that these cells (anti CD19 with CD137) migrated to bone marrow, expanded more than 1000 times and expressed at high level at least for 6 months functional CARs in chronic lymphocytic leukemia (CLL) patients. They showed a CD19-specific immune response in the blood and bone marrow, and complete remission in two out of three patients. However these engineered cells showed toxicity to B cells, plasma cells and resulted in hypogammaglobulinemia [100]. Song_et al showed later on that anti-FR $\alpha$ CAR showed strong antitumor activity in vitro but failed to be effective in an animal model. However, the CD137 (41BB) costimulatory motif in tandem (MOv19-BBל CAR) (anti-FR $\alpha$ CAR outfitted with CD137) proved an effective antitumor strategy against a xenograft metastatic human ovarian cancer model in immunodeficient mice [101]. Bretjens et al showed that autologous CD19 T-cells expressing both CD 28 and CD3 $\zeta$ signaling domains (CD19-CAR28 $\zeta$ ) rapidly trafficked to CD19+ chronic lymphocytic leukemia (CLL) or acute lymphoblastic leukemia (ALL) and evaluated the possibility of adaptive T cells therapy for clinical trial. They also showed that the engineered T cells retained ex vivo cytotoxicity at least 8 days after administration [102].

B-cell malignancies have been one of the most researched areas of CAR therapy because CD19 expressed on B-cells but not on progenitor cells is a clear target. Targeting CD19, though, has the potential to destroy all B-cells, not only malignancies. However, this is often considered an acceptable side effect. Recently, T-cells engineered with receptor tyrosine kinase-like orphan receptor 1 (ROR1) have been found to be effective for targeted therapy for B-CLL, mantle cell lymphoma and other ROR1 positive tumors. These cells do not target or show toxicity to mature B cells but do show toxicity to immature B cells (early stage cells or undifferentiated embryonic stem cells) and adipose tissue because of low level ROR1 expression [103]. 


\section{Conclusions}

Cell-directed therapy has been shown to be extremely versatile and effective in its applications especially in cancer therapy. The unique properties of cells allow them not only to selectively target tumors but also to actively infiltrate the tumor interstitium. They can also deliver a wide variety of payloads which can be combined in unique ways to create highly effective treatments. Moreover they can be programmed to home more effectively to tumors and/or to produce a treatment on site. In addition to all this, many cell types, themselves, may be naturally tumoricidal or may be encouraged to be tumoricidal. These properties demonstrate that cell-directed therapy is a useful and viable potential treatment for a variety of different cancers.

As demonstrated above, the field of cell-directed therapy is growing rapidly. Some of the more well-known cell types, such as those dealt with above, are being tested by multiple lab groups and a few have progressed to clinical trials. New tumor tropic cells are being tested as well and may become viable candidates for cell-directed therapy also. As new research continues and the understanding of the methods of tumor eradication and tumor tropism become clearer, cell-directed therapy will only continue to grow in strength as a potential treatment for cancer.

\section{Future Perspectives}

As the field of cytotherapeutic treatment of cancer continues to develop, several hurdles will need to be overcome. First, as discussed above, more rigorous definition of the fate of the delivery cells will need to be established and, relatedly, means of destroying the delivery cells after use will need to be developed and verified. Rigorous GMP facilities for clinical grade cells must also be developed. Since large numbers of well characterized cells are needed for a typical human dose, efficient means of generating these numbers without compromising cell quality must be developed. Also, the efficiency of tumor targeting by the delivery cells will need to be more rigorously defined and methods for increasing this targeting will continue to be developed. In addition, cell-directed therapies will need to identify means of procuring and engineering compatible delivery cells for human treatments that are both cost effective and safe. We expect to see a greater focus on these hurdles over the next decade as cytotherapy becomes a more widely accepted treatment possibility. As these hurdles are overcome, continuing research in this field will lead to increased clinical trials as promising methods are identified and controlled.

\section{Figures}

Figure 1: A: Nanoformulations penetrate tumors via extravasation through gaps between the endothelial cells. The delivery of nanoparticles is generally incomplete because of tumor heterogeneity and the presence of stromal cells. B: Tumor-homing cells (neural stem cells, 
mesenchymal stem cells, leucocytes and others) are tumor-homing and integrate with the tumor tissue. They are actively tumor-penetrating instead of merely following a concentration gradient. This enables enhanced tumor targeting and therapy.
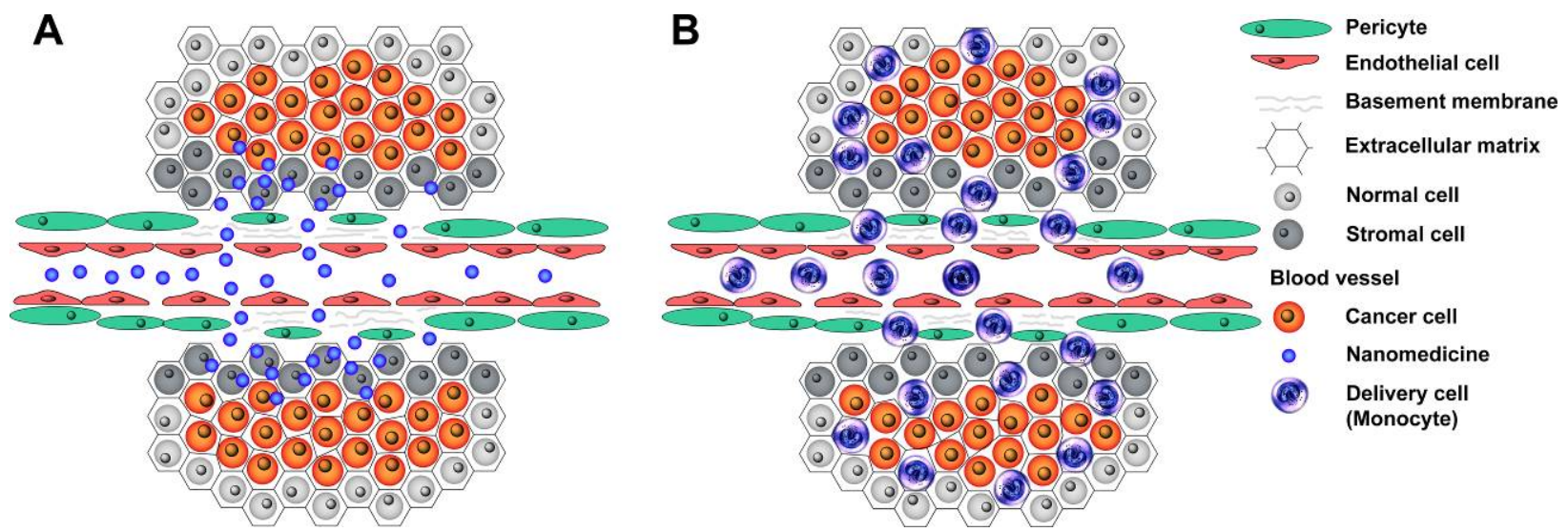

Figure 2: A: Bright-field image of C17.2 Neural Progenitor Cells (NPCs) after uptake of aminosiloxane-coated iron/iron oxide nanoparticles featuring chemically attached porphyrins (magnification 20x). The cells were stained with Prussian Blue and conterstained with nuclear fast red. Blue color indicates the presence of iron. B: Transmission electron microscopy image of (NPCs) after nanoparticle loading. It is clearly discernible that the iron/iron oxide nanoparticles form clusters within the cell. It is our paradigm that the nanoparticles are taken up via the endosomal pathway and remain attached to each other after escaping from (early) endosomes. C: Prussian blue stained NPCs loaded with iron/iron oxide nanoparticles in a tumor section of a black mouse bearing B16F10 melanoma cells. The melanoma cells are black. NPC containing transport cells have a blue color. One can clearly discern that the delivery cells are penetrating the tumor region. (The images used in Figure 1 were taken with permission (American Chemical Society, 2013) from reference 21).
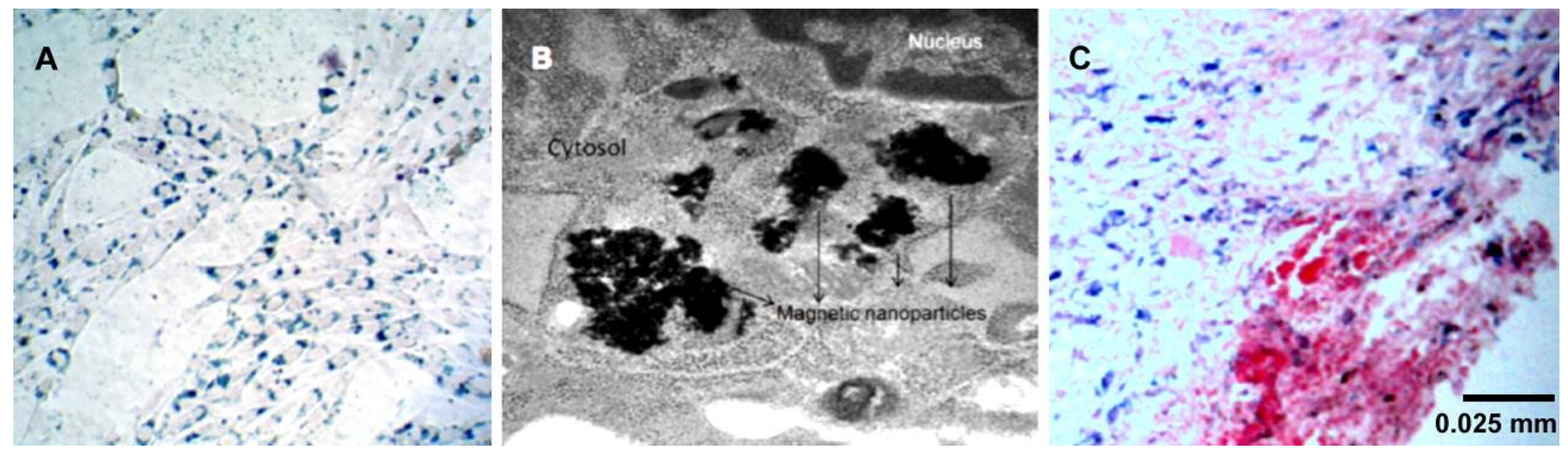
Figure 3 A: After uptake of dopamine-coated iron/iron oxide nanoparticles, monocyte/macrophage-like cells (Raw 264.7, Mo/Ma's) are injected interperitoneally into PAN02 (metastasizing pancreatic cancer) bearing black mice. The Mo/Ma cells that are capable of ameboid movement integrate with the PAN02 tumors. After they have reached the tumors, transport cells and neighboring cancer cells are subjected to magnetic hyperthermia, causing apoptosis. Hyperthermia is known to stimulate the immune response of the host to the tumor. B: The Hoechst nuclear counterstained section shows Mo/Ma labeled with PKH26 (red) in a pancreatic tumor, but not the adjacent pancreas. C: Cell-mediated magnetic hyperthermia was very effective when treating black mice bearing PAN02 tumors $(P<0.005$ for alternating magnetic field treatment versus all other groups). (The images used in Figure 2 were taken with permission from reference 83.)
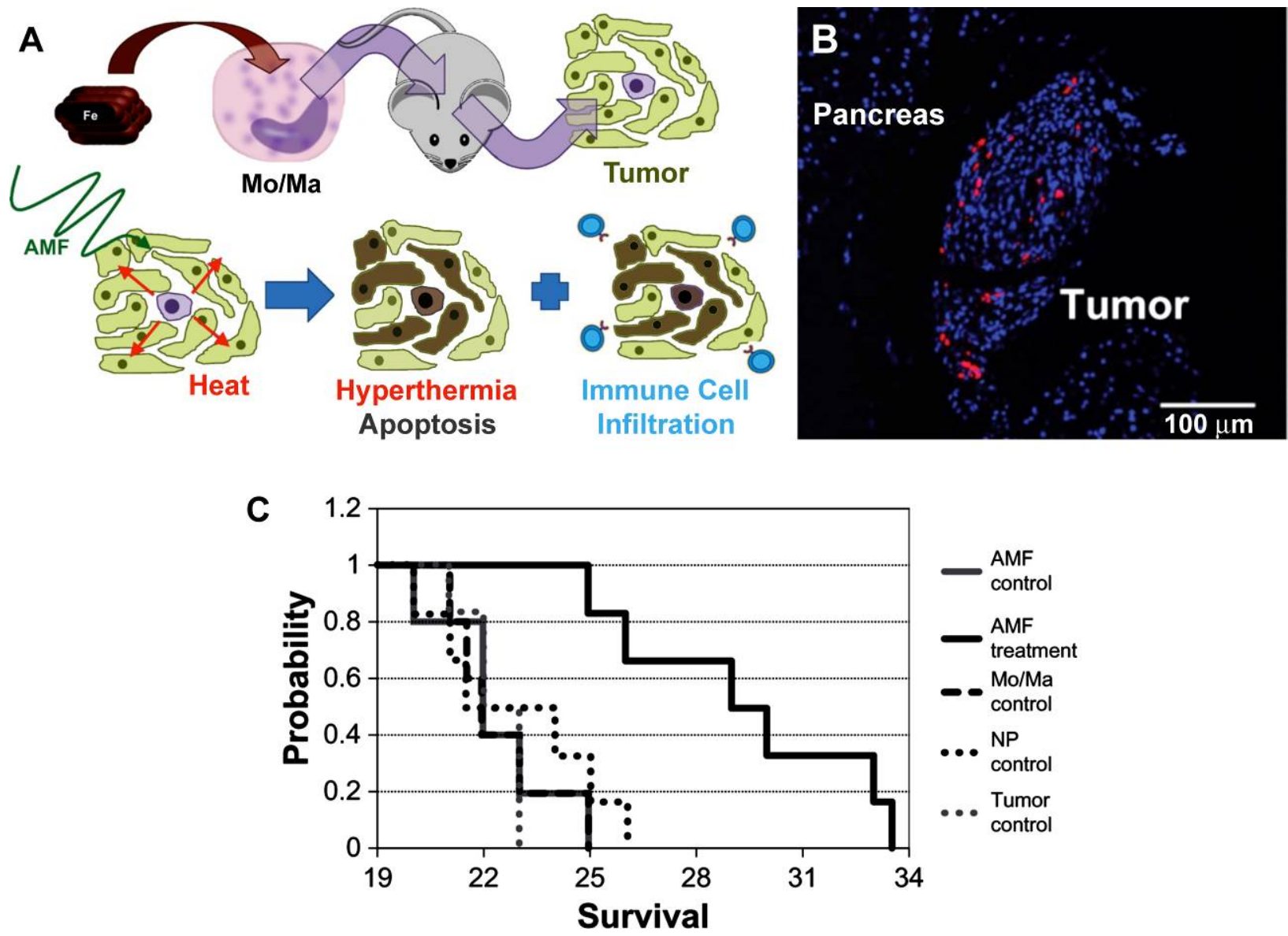


\section{Annotated References}

Aboody KS, Brown A, Rainov NG et al. Neural stem cells display extensive tropism for pathology in adult brain: evidence from intracranial gliomas. Proceedings of the National Academy of Sciences of the United States of America, 97(23), 12846-12851 (2000).

- Demonstrated for the first time that neural stem cells can infiltrate gliomas effectively

Aboody KS, Bush RA, Garcia E et al. Development of a tumor-selective approach to treat metastatic cancer. PloS one, 1, e23 (2006).

- Demonstrated the ability of neural stem cells to deliver a suicide gene to models of metastatic neuroblastoma

Basel MT, Balivada S, Wang H et al. Cell-delivered magnetic nanoparticles caused hyperthermiamediated increased survival in a murine pancreatic cancer model. International journal of nanomedicine, 7, 297-306 (2012).

- Demonstrated that monocytes loaded with magnetic nanoparticles can be used to treat locally metastatic pancreatic tumors with hyperthermia

Kucerova L, Altanerova V, Matuskova M, Tyciakova S, Altaner C. Adipose tissue-derived human mesenchymal stem cells mediated prodrug cancer gene therapy. Cancer research, 67(13), 6304-6313 (2007).

- Demonstrated the ability to engineer MSC with cytosine deaminase to target treatment to colon cancer

Ganta C, Chiyo D, Ayuzawa R et al. Rat umbilical cord stem cells completely abolish rat mammary carcinomas with no evidence of metastasis or recurrence 100 days posttumor cell inoculation. Cancer research, 69(5), 1815-1820 (2009).

- Demonstrated the significant tumoricidal nature of non-engineered mesenchymal stem cells

Ring KL, Tong LM, Balestra ME et al. Direct reprogramming of mouse and human fibroblasts into multipotent neural stem cells with a single factor. Cell stem cell, 11(1), 100-109 (2012).

- Demonstrated the ability to produce neural stem cells from fibroblasts by inserting Sox2

Studeny M, Marini FC, Champlin RE, Zompetta C, Fidler IJ, Andreeff M. Bone marrow-derived mesenchymal stem cells as vehicles for interferon-beta delivery into tumors. Cancer research, 62(13), 3603-3608 (2002).

- Demonstrated that mesenchymal stem cells can be used to produce therapeutic proteins at the site of the tumor

\section{References}


1. Fischbach MA, Bluestone JA, Lim WA: Cell-based therapeutics: the next pillar of medicine. Sci Transl Med 5(179), 179ps177 (2013).

2. Stem Cell Therapeutics for Cancer. Shah K (Ed.^^(Eds). John Wiley \& Sons, Inc., Hoboken, New Jersey (2013).

3. Lin G, Yang R, Banie L et al.: Effects of transplantation of adipose tissue-derived stem cells on prostate tumor. Prostate 70(10), 1066-1073 (2010).

4. Shinojima N, Hossain A, Takezaki T et al.: TGF-beta mediates homing of bone marrow-derived human mesenchymal stem cells to glioma stem cells. Cancer Res 73(7), 2333-2344 (2013).

5. Aboody KS, Brown A, Rainov NG et al.: Neural stem cells display extensive tropism for pathology in adult brain: evidence from intracranial gliomas. Proc Natl Acad Sci U S A 97(23), 12846-12851 (2000).

6. Aboody KS, Bush RA, Garcia E et al.: Development of a tumor-selective approach to treat metastatic cancer. PLoS One 1, e23 (2006).

7. Shimato S, Natsume A, Takeuchi H et al.: Human neural stem cells target and deliver therapeutic gene to experimental leptomeningeal medulloblastoma. Gene Ther 14(15), 1132-1142 (2007).

8. Wang C, Natsume A, Lee HJ et al.: Neural stem cell-based dual suicide gene delivery for metastatic brain tumors. Cancer Gene Ther 19(11), 796-801 (2012).

9. Bovenberg MS, Degeling MH, Tannous BA: Advances in stem cell therapy against gliomas. Trends Mol Med 19(5), 281-291 (2013).

10. Reitz M, Demestre $M$, Sedlacik J et al.: Intranasal delivery of neural stem/progenitor cells: a noninvasive passage to target intracerebral glioma. Stem Cells Transl Med 1(12), 866-873 (2012).

11. Gerecht-Nir S, Itskovitz-Eldor J: Human embryonic stem cells: a potential source for cellular therapy. Am J Transplant 4 Suppl 6, 51-57 (2004).

12. Balyasnikova IV, Ferguson SD, Han Y, Liu F, Lesniak MS: Therapeutic effect of neural stem cells expressing TRAIL and bortezomib in mice with glioma xenografts. Cancer Lett 310(2), 148-159 (2011).

13. Bagci-Onder T, Wakimoto H, Anderegg M, Cameron C, Shah K: A dual PI3K/mTOR inhibitor, PI103 , cooperates with stem cell-delivered TRAIL in experimental glioma models. Cancer Res 71(1), 154-163 (2011).

14. Van De Water JA, Bagci-Onder T, Agarwal AS et al.: Therapeutic stem cells expressing variants of EGFR-specific nanobodies have antitumor effects. Proc Natl Acad Sci U S A 109(41), 1664216647 (2012).

15. Frank RT, Edmiston M, Kendall SE et al.: Neural stem cells as a novel platform for tumor-specific delivery of therapeutic antibodies. PLoS One 4(12), e8314 (2009).

16. Thaci B, Ahmed AU, Ulasov IV et al.: Pharmacokinetic study of neural stem cell-based cell carrier for oncolytic virotherapy: targeted delivery of the therapeutic payload in an orthotopic brain tumor model. Cancer Gene Ther 19(6), 431-442 (2012).

17. Ahmed AU, Thaci B, Alexiades NG et al:: Neural stem cell-based cell carriers enhance therapeutic efficacy of an oncolytic adenovirus in an orthotopic mouse model of human glioblastoma. $\mathrm{Mol}$ Ther 19(9), 1714-1726 (2011).

18. Ahmed AU, Tyler MA, Thaci B et al.: A comparative study of neural and mesenchymal stem cellbased carriers for oncolytic adenovirus in a model of malignant glioma. Mol Pharm 8(5), 15591572 (2011).

19. Tobias AL, Thaci B, Auffinger B et al.: The timing of neural stem cell-based virotherapy is critical for optimal therapeutic efficacy when applied with radiation and chemotherapy for the treatment of glioblastoma. Stem Cells Transl Med 2(9), 655-666 (2013).

20. Schnarr K, Mooney R, Weng Y et al.: Gold nanoparticle-loaded neural stem cells for photothermal ablation of cancer. Adv Healthc Mater 2(7), 976-982 (2013). 
21. Rachakatla RS, Balivada S, Seo GM et al:: Attenuation of mouse melanoma by A/C magnetic field after delivery of bi-magnetic nanoparticles by neural progenitor cells. ACS Nano 4(12), 70937104 (2010).

22. Ring KL, Tong LM, Balestra ME et al.: Direct reprogramming of mouse and human fibroblasts into multipotent neural stem cells with a single factor. Cell Stem Cell 11(1), 100-109 (2012).

23. Yang J, Lam DH, Goh SS et al.: Tumor tropism of intravenously injected human-induced pluripotent stem cell-derived neural stem cells and their gene therapy application in a metastatic breast cancer model. Stem Cells 30(5), 1021-1029 (2012).

24. Chen C, Wang Y, Goh SS et al.: Inhibition of neuronal nitric oxide synthase activity promotes migration of human-induced pluripotent stem cell-derived neural stem cells toward cancer cells. J Neurochem 126(3), 318-330 (2013).

25. Center COHM: A Pilot Feasibility Study of Oral 5-Fluorocytosine and Genetically-Modified Neural Stem Cells Expressing E.Coli Cytosine Deaminase for Treatment of Recurrent High Grade Gliomas. 2013 Nov 19 NCT01172964, (2010).

26. Hori J, Ng TF, Shatos M, Klassen H, Streilein JW, Young MJ: Neural progenitor cells lack immunogenicity and resist destruction as allografts. Stem Cells 21(4), 405-416 (2003).

27. Michel-Monigadon D, Brachet P, Neveu I, Naveilhan P: Immunoregulatory properties of neural stem cells. Immunotherapy 3(4 Suppl), 39-41 (2011).

28. Xie J, Zhang H, Yea K, Lerner RA: Autocrine signaling based selection of combinatorial antibodies that transdifferentiate human stem cells. Proc Natl Acad Sci U S A 110(20), 8099-8104 (2013).

29. Studeny M, Marini FC, Champlin RE, Zompetta C, Fidler IJ, Andreeff M: Bone marrow-derived mesenchymal stem cells as vehicles for interferon-beta delivery into tumors. Cancer Res 62(13), 3603-3608 (2002).

30. Altaner C: Prodrug cancer gene therapy. Cancer Lett 270(2), 191-201 (2008).

31. Cihova M, Altanerova V, Altaner C: Stem cell based cancer gene therapy. Mol Pharm 8(5), 14801487 (2011).

32. Kucerova L, Matuskova M, Hlubinova K, Altanerova V, Altaner C: Tumor cell behaviour modulation by mesenchymal stromal cells. Mol Cancer 9, 129 (2010).

33. Rachakatla RS, Marini F, Weiss ML, Tamura M, Troyer D: Development of human umbilical cord matrix stem cell-based gene therapy for experimental lung tumors. Cancer Gene Ther 14(10), 828-835 (2007).

34. Rachakatla RS, Pyle MM, Ayuzawa R et al.: Combination treatment of human umbilical cord matrix stem cell-based interferon-beta gene therapy and 5-fluorouracil significantly reduces growth of metastatic human breast cancer in SCID mouse lungs. Cancer Invest 26(7), 662-670 (2008).

35. Ganta C, Chiyo D, Ayuzawa R et al.: Rat umbilical cord stem cells completely abolish rat mammary carcinomas with no evidence of metastasis or recurrence 100 days post-tumor cell inoculation. Cancer Res 69(5), 1815-1820 (2009).

36. Wang $M$, Yang $Y$, Yang $D$ et al.: The immunomodulatory activity of human umbilical cord bloodderived mesenchymal stem cells in vitro. Immunology 126(2), 220-232 (2009).

37. Nakamizo A, Marini F, Amano T et al.: Human bone marrow-derived mesenchymal stem cells in the treatment of gliomas. Cancer Res 65(8), 3307-3318 (2005).

38. Zou W, Zheng H, He TC, Chang J, Fu YX, Fan W: LIGHT delivery to tumors by mesenchymal stem cells mobilizes an effective antitumor immune response. Cancer Res 72(12), 2980-2989 (2012).

39. Zhu X, Su D, Xuan S et al.: Gene therapy of gastric cancer using LIGHT-secreting human umbilical cord blood-derived mesenchymal stem cells. Gastric Cancer 16(2), 155-166 (2013). 
40. Mader EK, Maeyama Y, Lin Y et al.: Mesenchymal stem cell carriers protect oncolytic measles viruses from antibody neutralization in an orthotopic ovarian cancer therapy model. Clin Cancer Res 15(23), 7246-7255 (2009).

41. Russell SJ, Peng KW: The utility of cells as vehicles for oncolytic virus therapies. Curr Opin Mol Ther 10(4), 380-386 (2008).

42. Sonabend AM, Ulasov IV, Tyler MA, Rivera AA, Mathis JM, Lesniak MS: Mesenchymal stem cells effectively deliver an oncolytic adenovirus to intracranial glioma. Stem Cells 26(3), 831-841 (2008).

43. Komarova S, Kawakami Y, Stoff-Khalili MA, Curiel DT, Pereboeva L: Mesenchymal progenitor cells as cellular vehicles for delivery of oncolytic adenoviruses. Mol Cancer Ther 5(3), 755-766 (2006).

44. Roger M, Clavreul A, Venier-Julienne MC et al.: Mesenchymal stem cells as cellular vehicles for delivery of nanoparticles to brain tumors. Biomaterials 31(32), 8393-8401 (2010).

45. Roger M, Clavreul A, Huynh NT et al.: Ferrociphenol lipid nanocapsule delivery by mesenchymal stromal cells in brain tumor therapy. Int J Pharm 423(1), 63-68 (2012).

46. Dembinski JL, Wilson SM, Spaeth EL et al.: Tumor stroma engraftment of gene-modified mesenchymal stem cells as anti-tumor therapy against ovarian cancer. Cytotherapy 15(1), 20-32 (2013).

47. Altanerova $\mathrm{V}$, Cihova $\mathrm{M}, \mathrm{Babic} \mathrm{M}$ et al.: Human adipose tissue-derived mesenchymal stem cells expressing yeast cytosinedeaminase::uracil phosphoribosyltransferase inhibit intracerebral rat glioblastoma. Int J Cancer 130(10), 2455-2463 (2012).

48. Cavarretta IT, Altanerova V, Matuskova M, Kucerova L, Culig Z, Altaner C: Adipose tissue-derived mesenchymal stem cells expressing prodrug-converting enzyme inhibit human prostate tumor growth. Mol Ther 18(1), 223-231 (2010).

49. Kucerova L, Matuskova M, Pastorakova A et al.: Cytosine deaminase expressing human mesenchymal stem cells mediated tumour regression in melanoma bearing mice. $J$ Gene Med 10(10), 1071-1082 (2008).

50. Altaner $\mathrm{C}$, Altanerova $\mathrm{V}$, Cihova $\mathrm{M}$ et al.: Complete regression of glioblastoma by mesenchymal stem cells mediated prodrug gene therapy simulating clinical therapeutic scenario. Int J Cancer 134(6), 1458-1465 (2014).

51. Grisendi G, Bussolari R, Cafarelli L et al.: Adipose-derived mesenchymal stem cells as stable source of tumor necrosis factor-related apoptosis-inducing ligand delivery for cancer therapy. Cancer Res 70(9), 3718-3729 (2010).

52. Khakoo AY, Pati S, Anderson SA et al.: Human mesenchymal stem cells exert potent antitumorigenic effects in a model of Kaposi's sarcoma. J Exp Med 203(5), 1235-1247 (2006).

53. Klopp AH, Gupta A, Spaeth E, Andreeff M, Marini F, 3rd: Concise review: Dissecting a discrepancy in the literature: do mesenchymal stem cells support or suppress tumor growth? Stem Cells 29(1), 11-19 (2011).

54. Kidd S, Spaeth E, Watson K et al.: Origins of the tumor microenvironment: quantitative assessment of adipose-derived and bone marrow-derived stroma. PLoS One 7(2), e30563 (2012).

55. Mishra PJ, Humeniuk R, Medina DJ et al.: Carcinoma-associated fibroblast-like differentiation of human mesenchymal stem cells. Cancer Res 68(11), 4331-4339 (2008).

56. Xu S, Menu E, De Becker A, Van Camp B, Vanderkerken K, Van Riet I: Bone marrow-derived mesenchymal stromal cells are attracted by multiple myeloma cell-produced chemokine CCL25 and favor myeloma cell growth in vitro and in vivo. Stem Cells 30(2), 266-279 (2012).

57. Li X, Ling W, Khan S, Yaccoby S: Therapeutic effects of intrabone and systemic mesenchymal stem cell cytotherapy on myeloma bone disease and tumor growth. $J$ Bone Miner Res 27(8), 1635-1648 (2012). 
58. Lamfers $\mathrm{M}$, Idema S, Van Milligen F et al.: Homing properties of adipose-derived stem cells to intracerebral glioma and the effects of adenovirus infection. Cancer Lett 274(1), 78-87 (2009).

59. Lee WY, Zhang T, Lau CP, Wang CC, Chan KM, Li G: Immortalized human fetal bone marrowderived mesenchymal stromal cell expressing suicide gene for anti-tumor therapy in vitro and in vivo. Cytotherapy 15(12), 1484-1497 (2013).

60. Roemeling-Van Rhijn M, De Klein A, Douben $\mathrm{H}$ et al.: Culture expansion induces non-tumorigenic aneuploidy in adipose tissue-derived mesenchymal stromal cells. Cytotherapy 15(11), 1352-1361 (2013).

61. Becker M, Muller CB, De Bastiani MA, Klamt F: The prognostic impact of tumor-associated macrophages and intra-tumoral apoptosis in non-small cell lung cancer. Histol Histopathol, (2013).

62. Chimal-Ramirez GK, Espinoza-Sanchez NA, Fuentes-Panana EM: Protumor activities of the immune response: insights in the mechanisms of immunological shift, oncotraining, and oncopromotion. J Oncol 2013, 835956 (2013).

63. Partecke LI, Gunther C, Hagemann S et al.: Induction of M2-macrophages by tumour cells and tumour growth promotion by $\mathrm{M} 2$-macrophages: a quid pro quo in pancreatic cancer.

Pancreatology 13(5), 508-516 (2013).

64. Baron-Bodo V, Doceur P, Lefebvre ML et al.: Anti-tumor properties of human-activated macrophages produced in large scale for clinical application. Immunobiology 210(2-4), 267-277 (2005).

65. Chen W, Liu J, Meng J et al.: Macrophage polarization induced by neuropeptide methionine enkephalin (MENK) promotes tumoricidal responses. Cancer Immunol Immunother 61(10), 17551768 (2012).

66. Nguyen HY, Vo BH, Nguyen LT et al.: Extracts of Crinum latifolium inhibit the cell viability of mouse lymphoma cell line EL4 and induce activation of anti-tumour activity of macrophages in vitro. J Ethnopharmacol 149(1), 75-83 (2013).

67. Rey-Giraud $\mathrm{F}$, Hafner $\mathrm{M}$, Ries $\mathrm{CH}$ : In vitro generation of monocyte-derived macrophages under serum-free conditions improves their tumor promoting functions. PLoS One 7(8), e42656 (2012).

68. De Palma M, Venneri MA, Galli R et al.: Tie2 identifies a hematopoietic lineage of proangiogenic monocytes required for tumor vessel formation and a mesenchymal population of pericyte progenitors. Cancer Cell 8(3), 211-226 (2005).

69. Du R, Lu KV, Petritsch C et al.: HIF1alpha induces the recruitment of bone marrow-derived vascular modulatory cells to regulate tumor angiogenesis and invasion. Cancer Cell 13(3), 206220 (2008).

70. De Palma M, Naldini L: Tie2-expressing monocytes (TEMs): novel targets and vehicles of anticancer therapy? Biochim Biophys Acta 1796(1), 5-10 (2009).

71. De Palma M, Mazzieri R, Politi LS et al.: Tumor-targeted interferon-alpha delivery by Tie2expressing monocytes inhibits tumor growth and metastasis. Cancer Cell 14(4), 299-311 (2008).

72. Russell JS, Brown JM: The irradiated tumor microenvironment: role of tumor-associated macrophages in vascular recovery. Front Physiol 4, 157 (2013).

73. Brugger W, Bross K, Frisch J et al.: Mobilization of peripheral blood progenitor cells by sequential administration of interleukin-3 and granulocyte-macrophage colony-stimulating factor following polychemotherapy with etoposide, ifosfamide, and cisplatin. Blood 79(5), 1193-1200 (1992).

74. Krosl G, Korbelik M, Dougherty GJ: Induction of immune cell infiltration into murine SCCVII tumour by photofrin-based photodynamic therapy. Br J Cancer 71(3), 549-555 (1995).

75. Ma Y, Adjemian S, Mattarollo SR et al.: Anticancer chemotherapy-induced intratumoral recruitment and differentiation of antigen-presenting cells. Immunity 38(4), 729-741 (2013). 
76. Skivka LM, Gorobets OB, Kutsenok VV et al.: 5-aminolevulinic acid mediated photodynamic therapy of Lewis lung carcinoma: a role of tumor infiltration with different cells of immune system. Exp Oncol 26(4), 312-315 (2004).

77. Schioppa T, Uranchimeg B, Saccani A et al.: Regulation of the chemokine receptor CXCR4 by hypoxia. J Exp Med 198(9), 1391-1402 (2003).

78. Kean TJ, Duesler L, Young RG et al.: Development of a peptide-targeted, myocardial ischemiahoming, mesenchymal stem cell. J Drug Target 20(1), 23-32 (2012).

79. Sharma A, Kapoor P, Gautam A et al.: Computational approach for designing tumor homing peptides. Sci Rep 3, 1607 (2013).

80. Muthana $\mathrm{M}$, Scott SD, Farrow $\mathrm{N}$ et al.: A novel magnetic approach to enhance the efficacy of cell-based gene therapies. Gene Ther 15(12), 902-910 (2008).

81. Choi MR, Stanton-Maxey KJ, Stanley JK et al.: A cellular Trojan Horse for delivery of therapeutic nanoparticles into tumors. Nano Lett 7(12), 3759-3765 (2007).

82. Baek SK, Makkouk AR, Krasieva T, Sun CH, Madsen SJ, Hirschberg H: Photothermal treatment of glioma; an in vitro study of macrophage-mediated delivery of gold nanoshells. J Neurooncol 104(2), 439-448 (2011).

83. Basel MT, Balivada S, Wang $\mathrm{H}$ et al.: Cell-delivered magnetic nanoparticles caused hyperthermiamediated increased survival in a murine pancreatic cancer model. Int J Nanomedicine 7, 297-306 (2012).

84. Seo GM, Rachakatla RS, Balivada $S$ et al.: A self-contained enzyme activating prodrug cytotherapy for preclinical melanoma. Mol Biol Rep 39(1), 157-165 (2012).

85. Basel MT, Balivada S, Shrestha TB et al.: A cell-delivered and cell-activated SN38-dextran prodrug increases survival in a murine disseminated pancreatic cancer model. Small 8(6), 913920 (2012).

86. Bunuales M, Garcia-Aragoncillo E, Casado R et al.: Evaluation of monocytes as carriers for armed oncolytic adenoviruses in murine and Syrian hamster models of cancer. Hum Gene Ther 23(12), 1258-1268 (2012).

87. Muthana M, Giannoudis A, Scott SD et al.: Use of macrophages to target therapeutic adenovirus to human prostate tumors. Cancer Res 71(5), 1805-1815 (2011).

88. Choi MR, Bardhan R, Stanton-Maxey KJ et al.: Delivery of nanoparticles to brain metastases of breast cancer using a cellular Trojan horse. Cancer Nanotechnol 3(1-6), 47-54 (2012).

89. Matsui M, Shimizu Y, Kodera Y, Kondo E, Ikehara Y, Nakanishi H: Targeted delivery of oligomannose-coated liposome to the omental micrometastasis by peritoneal macrophages from patients with gastric cancer. Cancer Sci 101(7), 1670-1677 (2010).

90. Choi J, Kim HY, Ju EJ et al.: Use of macrophages to deliver therapeutic and imaging contrast agents to tumors. Biomaterials 33(16), 4195-4203 (2012).

91. Batrakova EV, Li S, Reynolds AD et al.: A macrophage-nanozyme delivery system for Parkinson's disease. Bioconjug Chem 18(5), 1498-1506 (2007).

92. Gonzalez-Mejia ME, Doseff Al: Regulation of monocytes and macrophages cell fate. Front Biosci (Landmark Ed) 14, 2413-2431 (2009).

93. Zhang JX, Kang CS, Shi L, Zhao P, Liu N, You YP: Use of thymidine kinase gene-modified endothelial progenitor cells as a vector targeting angiogenesis in glioma gene therapy. Oncology 78(2), 94-102 (2010).

94. Eisenstein S, Coakley BA, Briley-Saebo K et al.: Myeloid-derived suppressor cells as a vehicle for tumor-specific oncolytic viral therapy. Cancer Res 73(16), 5003-5015 (2013).

95. Becker ML, Near R, Mudgett-Hunter $M$ et al.: Expression of a hybrid immunoglobulin-T cell receptor protein in transgenic mice. Cell 58(5), 911-921 (1989). 
96. Gross G, Gorochov G, Waks T, Eshhar Z: Generation of effector T cells expressing chimeric T cell receptor with antibody type-specificity. Transplant Proc 21(1 Pt 1), 127-130 (1989).

97. Gross G, Waks T, Eshhar Z: Expression of immunoglobulin-T-cell receptor chimeric molecules as functional receptors with antibody-type specificity. Proc Natl Acad Sci U S A 86(24), 1002410028 (1989).

98. Kuwana $\mathrm{Y}$, Asakura $\mathrm{Y}$, Utsunomiya $\mathrm{N}$ et al.: Expression of chimeric receptor composed of immunoglobulin-derived $\mathrm{V}$ regions and T-cell receptor-derived $\mathrm{C}$ regions. Biochem Biophys Res Commun 149(3), 960-968 (1987).

99. Milone MC, Fish JD, Carpenito $C$ et al.: Chimeric receptors containing CD137 signal transduction domains mediate enhanced survival of T cells and increased antileukemic efficacy in vivo. Mol Ther 17(8), 1453-1464 (2009).

100. Kalos M, Levine BL, Porter DL et al.: T cells with chimeric antigen receptors have potent antitumor effects and can establish memory in patients with advanced leukemia. Sci Trans/ Med 3(95), 95 ra73 (2011).

101. Song DG, Ye $\mathrm{Q}$, Carpenito $\mathrm{C}$ et al.: In vivo persistence, tumor localization, and antitumor activity of CAR-engineered T cells is enhanced by costimulatory signaling through CD137 (4-1BB). Cancer Res 71(13), 4617-4627 (2011).

102. Brentjens RJ, Riviere I, Park JH et al.: Safety and persistence of adoptively transferred autologous CD19-targeted T cells in patients with relapsed or chemotherapy refractory B-cell leukemias. Blood 118(18), 4817-4828 (2011).

103. Hudecek M, Schmitt TM, Baskar S et al.: The B-cell tumor-associated antigen ROR1 can be targeted with T cells modified to express a ROR1-specific chimeric antigen receptor. Blood 116(22), 4532-4541 (2010). 